\title{
Aceitação de dietas em ambiente hospitalar
}

\author{
Acceptance of hospital food
}

Anete Araújo de SOUSA ${ }^{1}$

Mariana de Souza GLORIA'

Thalita Schütz CARDOSO'

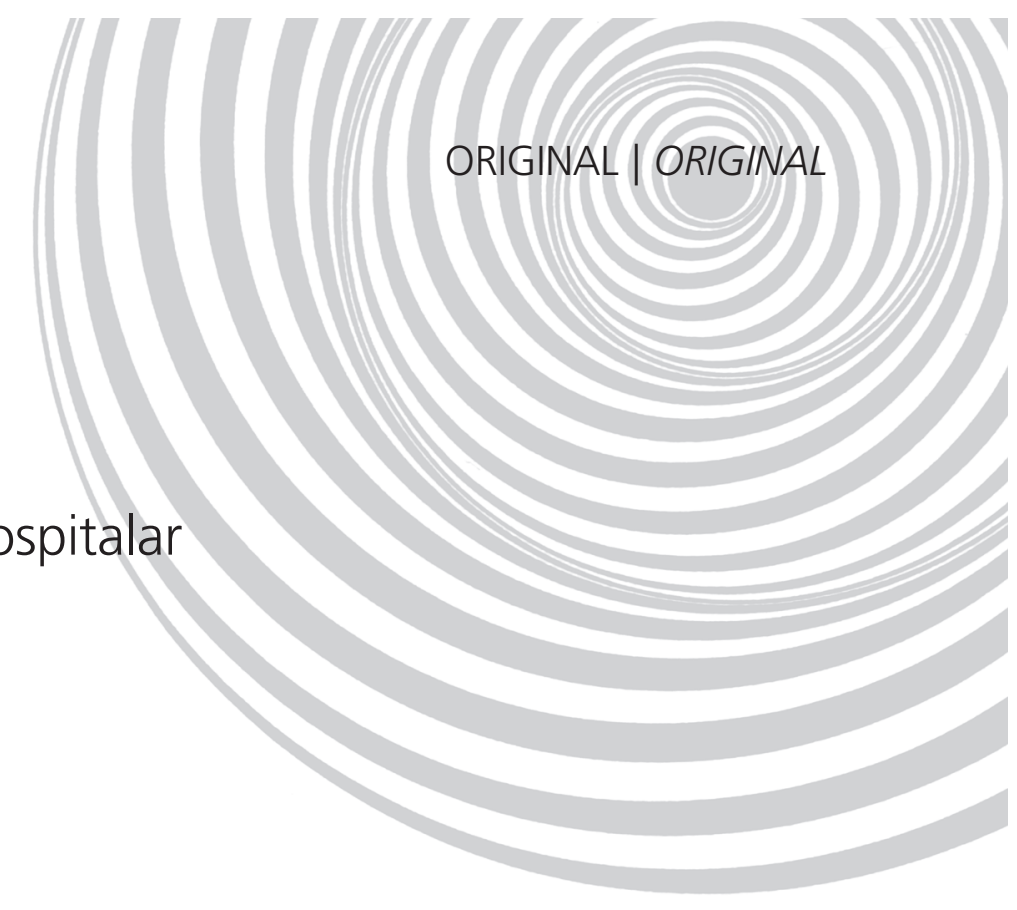

RE S U M O

\section{Objetivo}

O objetivo do estudo foi avaliar a aceitação de dietas de pacientes internados em um hospital público do município de Florianópolis, Santa Catarina.

\section{Métodos}

Realizou-se análise dos restos alimentares de pacientes internados em unidades de internação médica durante 15 dias, por meio do índice resto-ingestão. O índice foi determinado por tipos de dieta, classificadas em normal, especial com sal e especial sem sal. Enquetes de satisfação com pacientes avaliaram os fatores que influenciam a aceitação das refeições.

\section{Resultados}

O peso total distribuído correspondente a 1877 refeições foi de $868 \mathrm{~kg}$ e o peso dos restos totalizou $313 \mathrm{~kg}$. Foi encontrado um índice resto-ingestão de 36,09\% e um retorno de 53,68\%, 33,99\% e 33,84\% de dietas especiais com sal, normais e especiais sem sal respectivamente. A variedade, a quantidade e a apresentação das refeições foram avaliadas de forma positiva. A temperatura e o uso de temperos foram os aspectos de menor satisfação, representando $43,00 \%$ e $34,30 \%$, respectivamente, para os critérios de regular a ruim. O índice resto-ingestão encontrado (36,09\%) estava acima do aceitável para uma população enferma $(20,00 \%)$, demonstrando uma perda considerável de alimentos.

\section{Conclusão}

A partir dos resultados, observou-se a necessidade de avaliar os processos envolvidos na produção dessas refeições e de elaborar estratégias que estimulem a ingestão alimentar do paciente.

Termos de indexação: Aceitação pelo paciente de cuidados de saúde. Alimentação coletiva. Dieta. Serviço Hospitalar de Nutrição.

\section{A B S T R A C T}

\section{Objective}

This study assessed patients' acceptance of hospital foods in a public hospital located in Florianópolis, Santa Catarina, Brazil.

\footnotetext{
1 Universidade Federal de Santa Catarina, Centro de Ciências da Saúde, Curso de Nutrição, Departamento de Nutrição. Caixa Postal 476, 88040-900, Trindade, Florianópolis, SC, Brasil. Correspondência para/Correspondence to: A.A. SOUSA. E-mail: <anete@ccs.ufsc.br>.
} 
288 | A.A. SOUSA et al.

\section{Methods}

The leftover-ingestion index was used to analyze the volume of leftovers of hospitalized patients during 15 days. The index was determined by diet type: normal, special with salt and special without salt. Satisfaction questionnaires were administered to the patients and the factors that influence food acceptance were assessed.

\section{Results}

The total weight of 1,877 meals was $868 \mathrm{~kg}$ and the weight of the leftovers was $313 \mathrm{~kg}$, thus a leftoveringestion index of $36.09 \%$. The percentage of leftovers of the special diets with salt, normal diets and special diets without salt were 53.68\%, 33.99\% and 33.84\%, respectively. The patients were satisfied with the variety, amount and presentation of the meals but complained about the temperature and seasonings, with ratings of $43.00 \%$ and $34.30 \%$, respectively. The leftover-ingestion index (36.09\%) was above the acceptable index (20.00\%) for a diseased population, indicating a considerable waste of food.

\section{Conclusion}

The results show that it is necessary to assess the meal production processes and develop strategies that encourage the patients to eat.

Indexing terms: Patient's acceptance of health care. Collective food. Diet. Food service, hospital.

\section{N T R O D U ÇÃ O}

A preocupação com indicadores do estado nutricional do indivíduo hospitalizado tem sido crescente, uma vez que a desnutrição intra-hospitalar continua sendo a causa mais frequente do aumento da morbimortalidade na internação, atingindo entre $30 \%$ e $50 \%$ das pessoas hospitalizadas de todas as idades ${ }^{1-3}$.

Garcia ${ }^{4}$ argumenta que, entre os fatores causais atribuídos à desnutrição hospitalar, a alimentação é considerada um fator circunstancial em razão das mudanças alimentares, troca de hábitos e horários alimentares.

Além disso, mais da metade dos pacientes hospitalizados depende da unidade responsável pela alimentação para obtenção de sua única fonte de nutrição. Destaca-se, ainda, que pacientes internados por um período abaixo de cinco dias podem sofrer menos com a redução do consumo nutricional. No entanto, pacientes severamente doentes, em risco nutricional e internados por longo período, podem tornar-se desnutridos, aumentando-se as complicações, o período de internação e, consequentemente, os custos ${ }^{5}$.

Nonino-Borges et al. ${ }^{6}$ destacam a complexidade do trabalho da instituição hospitalar, ao compatibilizar a oferta de refeições com as necessidades nutricionais e terapêuticas dos pacien- tes, as responsabilidades econômico-administrativas e a programação e execução das refeições.

Estudos têm demonstrado uma avaliação insuficiente do estado nutricional e da capacidade de ingestão alimentar dos pacientes, o que resulta em inadequação de proteínas e calorias, bem como na produção superestimada de alimentos, o que, por sua vez, acarreta desperdício ${ }^{7-10}$. A ingestão alimentar insuficiente é sempre atribuída, primeiramente, aos aspectos clínicos. No entanto, outros estudos observaram que os pacientes não ingerem boa parte da alimentação que lhes é oferecida em razão não apenas da doença, da falta de apetite e das alterações do paladar, mas também da mudança de hábitos e da insatisfação com as preparações e o ambiente hospitalar ${ }^{11-13}$. Além disso, a aceitação da alimentação também tem sido relacionada com o tipo de atendimento prestado ${ }^{14-16}$

Observa-se, ainda, que a alimentação hospitalar é alvo de críticas e rejeições por parte dos pacientes e da população em geral. A comida de hospital é comumente percebida como insossa, sem gosto, fria, servida cedo e ainda com conotações de permissão e proibição ${ }^{17}$.

Parte dessas observações pode ser analisada, uma vez que a alimentação não pode se restringir ao processo que vai da recepção da matéria-prima ao garfo ${ }^{10}$ e nem como fonte de 
nutrição que promove energia, vitalidade e saúde. $\mathrm{O}$ ato alimentar é parte da identidade pessoal, cultural e social dos indivíduos e é construído nas relações sociais entre amigos, familiares e da sociedade como um todo. O alimento traz uma gama de significados simbólicos que expressam e criam as relações entre os seres humanos e o ambiente natural em que vivem ${ }^{18}$.

Poulain et al. ${ }^{19}$ consideram que um alimento, para ser capaz de manter a vida, não deve ter somente qualidades nutricionais, expressas pelas quantidades de glicídios, lipídeos, proteínas, vitaminas e minerais. É necessário que ele seja conhecido e/ou aceito pelo indivíduo e pelo grupo social, e que a alimentação apresente quatro funções essenciais: nutricional, higiênica, hedônica e convivial. Assim, aspectos como o gosto, a cor, a forma, o aroma e a textura, além da temperatura das refeições, o horário de distribuição, o ambiente onde se dá a refeição, dentre outros elementos, são componentes que precisam ser considerados na abordagem nutricional ${ }^{20}$.

Os estudos de determinação do resto-ingestão nas refeições hospitalares são pouco referidos na literatura. No Brasil, o estudo de Nonino-Borges et al. ${ }^{6}$ relacionou desperdício e custo alimentar. Estudos internacionais como os de Barton et al. ${ }^{8}$ e Kondrup ${ }^{10}$ avaliaram a ingestão das refeições com objetivo de observar se as necessidades nutricionais dos pacientes foram atendidas. Almdal et al.7, na Dinamarca, estudaram o consumo alimentar de pacientes durante três dias em um hospital universitário e obtiveram não só a avaliação das necessidades nutricionais de pacientes, como também o desperdício gerado com o retorno das refeições.

Diante das considerações anteriores, o objetivo deste estudo foi avaliar a aceitação de dietas de pacientes internados em um hospital público no município de Florianópolis. Como parte de um projeto de implementação de ações humanizadoras na alimentação hospitalar, essa etapa teve como finalidade subsidiar a construção de estratégias que possibilitem o aumento do consumo alimentar no hospital.

\section{M É T O D OS}

O estudo, que utilizou uma abordagem exploratória, foi desenvolvido em um hospital público, referência para a Política Nacional de Humanização, no município de Florianópolis. O hospital conta com 263 leitos, 100\% Sistema Único de Saúde (SUS), distribuídos entre Unidades de Internação (UI) médicas, cirúrgicas, pediátrica e maternidade, além da emergência e unidade de terapia intensiva.

A Unidade de Alimentação e Nutrição (UAN) produz 1736 refeições/dia, além de 913 mamadeiras, 185 complementos e 379 dietas enterais, mensalmente, no setor de lactário, bem como 1518 mamadeiras para a neonatologia, e responsabiliza-se, ainda, pela aquisição e recepção de gêneros alimentícios para a produção de refeições da creche do grêmio de servidores.

As refeições da dieta normal e das dietas especiais são produzidas nos setores da cozinha geral e da cozinha dietética respectivamente. Os cardápios são padronizados e contam com oito cardápios (dois meses) para cozinha geral e dois cardápios para a cozinha dietética (15 dias). 0 porcionamento das refeições aos pacientes é realizado de forma centralizada, em recipientes térmicos.

Para os propósitos do estudo, a avaliação da aceitação das dietas foi realizada em diferentes momentos, estabelecendo-se as seguintes etapas:

Seleção das Unidades de Internação: as unidades de internação do hospital selecionadas para o estudo foram as Unidades de Internação Médicas (UIM), com base em critérios de tempo de internação dos pacientes e maior variedade de dietas. As UIM são distribuídas em UIM I, II e III e apresentam, em média, 30 leitos por unidade. O tempo de internação médio de pacientes nessas unidades é de 13 dias.

Determinação do índice de resto-ingestão: a avaliação da aceitação dos pacientes foi determinada quantitativamente, pelo Índice de Resto-ingestão (IR), referido por Gandra ${ }^{21}$, que estabelece a relação percentual entre o Peso da Refeição 
rejeitada (PR) e o Peso da Refeição Distribuída (PRD), representada pela fórmula: $I R=P R \times 100 /$ PRD. As refeições avaliadas foram o almoço e o jantar das dietas normais, especiais e especiais hipossódicas. A coleta de dados para o cálculo do IR foi realizada durante quinze dias úteis (três semanas), a partir dos seguintes procedimentos:

Determinação do peso das refeições distribuídas (PRD): inicialmente, foi determinado o peso de utensílios utilizados para o porcionamento das preparações. Foram pesados (trinta) recipientes térmicos, cinco sopeiras e oito embalagens descartáveis, obtendo-se o valor do peso médio desses recipientes. Posteriormente, esses utensílios foram pesados com as refeições. O valor total do peso das refeições, deduzindo-se o peso dos utensílios, permitiu a determinação do PRD. Utilizou-se uma balança digital da marca Filizola com sensibilidade de $5 \mathrm{~g}$, capacidade máxima de $30 \mathrm{~kg}$ e mínima de $125 \mathrm{~g}$.

Determinação do peso dos restos das refeições (PR): os restos das refeições provenientes das UIM foram acondicionados em recipientes específicos para cada grupo de dietas: normal, especial com sal e especial sem sal. As sobremesas e saladas não foram avaliadas neste estudo. Separou-se, ainda, a parte não comestível de alguns alimentos, como os ossos (frango e bisteca suína) e os descartáveis (copos e guardanapos). O valor total do peso dos restos, deduzindo-se o peso da parte não comestível, permitiu a determinação do PR. Utilizou-se uma balança tipo plataforma, modelo PLE, com sensibilidade de $100 \mathrm{~g}$ e capacidade máxima de $300 \mathrm{~kg}$ e mínima de $2 \mathrm{~kg}$.

Avaliação dos fatores que influenciam na aceitação das refeições: a avaliação da aceitação foi realizada, ainda, com pacientes internados nas UIM há mais de quatro dias, através de enquetes de satisfação. Ao todo foram entrevistados 35 pacientes. $O$ instrumento utilizado foi um formulário, adaptado de Proença et al. ${ }^{20}$ Os indicadores avaliados foram: variedade do cardápio, apresentação, temperatura, tempero e quantidade da refeição. Como parte de um projeto de pesquisa intitulado "Aceitação da dieta hipossódica de pacientes hospitalizados" e aprovado pelo Comitê de Ética em Pesquisa com Seres Humanos (CEPSH) da Universidade Federal de Santa Catarina, protocolo n²09/2007, todos os participantes assinaram o termo de consentimento livre e esclarecido, antes de sua inclusão na amostra.

Os dados obtidos foram organizados e tabulados pelo programa Microsoft Office Excel, e a avaliação dos resultados foi feita por método estatístico descritivo. Para a contabilização e análise dos dados coletados, foi utilizado o programa Microsoft Office Excel 2007. Para a comparação dos valores obtidos entre a refeição do almoço e do jantar e dos tipos de dietas avaliadas (normal, com sal e sem sal), utilizou-se o teste de Mann-Whitney, com auxilio do software Statistical Package for the Social Sciences (SPSS) 16.0.

\section{RESULTADOS}

\section{O índice de resto-ingestão}

Na determinação do peso das dietas avaliadas (normais, especiais com sal e especiais sem sal), observou-se que as dietas especiais com sal apresentam, em sua maioria, modificações em sua consistência (branda, pastosa e líquida) e em sua composição (dieta para diabetes mellitus, hipolipídica, dieta pobre em $\mathrm{K}$ e hipofermentativa). Ao contrário, nas dietas especiais sem sal, a consistência foi predominantemente normal.

Foi determinado o peso de 1877 refeições distribuídas nas unidades de internação médicas: 937 foram refeições do almoço e 940 do jantar.

A Tabela 1 apresenta o peso das refeições fornecidas e dos restos alimentares em quilogramas e a relação percentual de resto-ingestão. A Tabela 2 apresenta o peso fornecido e rejeitado e a relação percentual de resto-ingestão dos diferentes tipos de dietas.

A comparação dos pesos $(\mathrm{kg})$ observados no almoço e no jantar nos dias analisados indicou não haver diferença significativa $(p=0,8517)$, su- 
gerindo que essas refeições apresentam indicadores de desperdício aproximados.

O confronto dos pesos obtidos nas diferentes dietas nos dias examinados indicou haver diferença significativa entre os tipos de dieta (Tabela 3). Os resultados mostraram que a dieta com sal resulta em maior percentual de restos.

Tabela 1. Peso das refeições fornecidas e dos restos alimentares (em kg) e a relação percentual de resto-ingestão. Florianópolis (SC), 2008.

\begin{tabular}{llll}
\hline Refeição & PRD $(\mathrm{kg})$ & PR $(\mathrm{kg})$ & \% do resto \\
\hline Almoço & 430,63 & 157,39 & 36,55 \\
Jantar & 438,30 & 156,18 & 35,63 \\
\hline Total & 868,93 & 313,56 & 36,09 \\
\hline
\end{tabular}

PRD: peso da refeição distribuída; PR: peso da refeição.

Tabela 2. Peso das dietas fornecidas e de restos alimentares (em $\mathrm{kg}$ ) e a relação percentual de resto-ingestão dos diferentes tipos de dietas. Florianópolis (SC), 2008.

\begin{tabular}{lccc}
\hline Tipos de dietas & Fornecido $(\mathrm{kg})$ & Resto $(\mathrm{kg})$ & $\%$ do resto \\
\hline Normal & 313,27 & 106,48 & 33,99 \\
Especial & & & \\
Com sal & 95,93 & 51,50 & 53,68 \\
Sem sal & 459,73 & 155,58 & 33,84 \\
\hline Total & 868,93 & 313,56 & 36,09 \\
\hline
\end{tabular}

\section{Fatores que influenciam na aceitação das refeições}

Os pacientes entrevistados são mulheres (17) e homens (18). A maioria recebeu dieta especial hipossódica, representando 74,30\% (26) do total, além de pacientes com dieta normal, 14,28\% (5) e especial com sal, $11,42 \%$ (4). A avaliação percentual dos indicadores referentes à variedade, apresentação, temperatura, tempero e quantidade da alimentação recebida no almoço e no jantar é apresentada na Tabela 4.

A variedade, a quantidade e a apresentação das refeições foram avaliadas de forma positiva pelos pacientes. Em relação à temperatura e ao uso de temperos, para os critérios de ruim a regular, as avaliações foram de 43,00\% e 34,30\%, respectivamente.

\section{I S C U S S Ã O}

O estudo teve como objetivo avaliar a aceitação de dietas oferecidas aos pacientes das unidades de internação médicas de um hospital público do município de Florianópolis, por meio da determinação do índice de resto-ingestão e de enquetes de satisfação.

Tabela 3. Comparação entre os valores encontrados de índices de resto-ingestão de acordo com o tipo de dieta oferecida. Florianópolis (SC), 2008

\begin{tabular}{lll}
\hline Dietas & Teste $^{*}$ & \multicolumn{1}{c}{ Comparação entre dietas } \\
\hline Normal $x$ especial com sal & $p<0,001$ & Há mais restos nas dietas com sal \\
Normal $x$ especial sem sal & $p=0,8925$ & Não há diferença de restos entre as duas dietas \\
Especial com sal x especial sem sal & $p<0,001$ & Há mais restos nas dietas com sal \\
\hline
\end{tabular}

${ }^{*}$ Teste de Mann-Whitney.

Tabela 4. Enquetes de satisfação no hospital analisado. Florianópolis (SC), 2008.

\begin{tabular}{|c|c|c|c|c|c|c|c|c|c|c|}
\hline \multirow{2}{*}{ Enquete } & \multicolumn{2}{|c|}{ Bom } & \multicolumn{2}{|c|}{ Regular } & \multicolumn{2}{|c|}{ Ruim } & \multicolumn{2}{|c|}{ Não respondeu } & \multicolumn{2}{|c|}{ Total } \\
\hline & $n$ & $\%$ & $\mathrm{n}$ & $\%$ & $\mathrm{n}$ & $\%$ & $n$ & $\%$ & $\mathrm{n}$ & $\%$ \\
\hline Variedade & 28 & 80,0 & 5 & 14,3 & 2 & 5,7 & 0 & 0 & 35 & 100,0 \\
\hline Apresentação & 30 & 85,7 & 4 & 11,3 & 1 & 3,0 & 0 & 0 & 35 & 100,0 \\
\hline Temperatura & 20 & 57,0 & 8 & 23,0 & 7 & 20,0 & 0 & 0 & 35 & 100,0 \\
\hline Tempero & 22 & 62,8 & 8 & 23,0 & 4 & 11,3 & 1 & 3,0 & 35 & 100,0 \\
\hline Quantidade & 28 & 80,0 & 2 & 5,7 & 4 & 11,3 & 1 & 3,0 & 35 & 100,0 \\
\hline
\end{tabular}


O índice de resto-ingestão referente a um período de quinze dias úteis apresentou uma taxa média de 36,09\% (36,55\% no almoço e 35,63\% no jantar). Resultados semelhantes foram obtidos em alguns estudos. Nonino-Borges et al. ${ }^{6}$ encontraram um índice de resto-ingestão de 33,00\% para o almoço e de $35,00 \%$ para o jantar. Já no estudo de Almdal et al. ${ }^{7}$, observou-se um retorno de $10300 \mathrm{~kg}$ de alimentos não consumidos sobre $26500 \mathrm{~kg}$ de alimentos fornecidos, o que equivale a um índice de resto-ingestão de 38,80\%.

Castro et al. ${ }^{22}$ destacam que, quando os valores de resto-ingestão estão acima de $20 \%$ em coletividades enfermas, pressupõe-se inadequação no planejamento e na execução dos cardápios. Outros estudos ${ }^{7,8}$ argumentam que o índice de resto-ingestão elevado pode ser decorrente de falha no porcionamento, preparações incompatíveis com o padrão/hábito dos pacientes, má apresentação das preparações, preparação de dietas a pacientes em jejum ou que receberam alta, prescrição de dietas gerais a pacientes com problemas de dentição ou com níveis de consciência alterados e baixa assistência aos pacientes que não conseguem se alimentar direito.

Dupertuis et al. ${ }^{14}$, ao analisarem a adequação das refeições em hospitais, demonstraram que, apesar da oferta suficiente de alimentos, a maioria dos pacientes hospitalizados não teve suas necessidades nutricionais atendidas. A ingestão insuficiente era comumente atribuída a causas que excediam as doenças; dessa forma, o estudo recomenda a necessidade de melhorar os serviços ligados às refeições.

Zazzo \& Puissant ${ }^{23}$ observaram que os problemas relacionados ao consumo estavam ligados à doença ou ao tratamento (32,10\% dos casos), à organização da unidade de produção e das unidades de internação ou a uma má integração entre esses setores ( $53,60 \%$ dos casos), entre outros problemas ( $14,30 \%$ dos casos).

O índice de resto-ingestão elevado no presente estudo pode estar relacionado ao tipo de público atendido pelas UIM, que, na sua maioria, é composto por idosos que apresentam condições próprias que podem interferir no seu estado nutricional e alterar sua ingestão alimentar. Alguns desses condicionantes são devidos às alterações fisiológicas próprias do envelhecimento, como alterações sensoriais, redução da sensibilidade por gostos primários, diminuição da salivação e alteração da capacidade mastigatória. Podem também ser influenciados pelas enfermidades e/ou fatores psicossociais, tais como perda do cônjuge, depressão, isolamento social, pobreza, capacidade de deslocamento, capacidade cognitiva, e pelos fatores associados à própria enfermidade, como inapetência e náuseas ${ }^{24}$.

Kondrup ${ }^{10}$ argumenta ainda que outros aspectos interferem na aceitação de uma refeição: desde a recepção da matéria-prima até a distribuição das preparações, que envolvem o ambiente onde se faz a refeição e o acompanhamento do consumo alimentar. Destaca ainda que aspectos nutricionais e políticas voltadas para a melhoria da qualidade da assistência alimentar são importantes, no entanto os aspectos culturais relacionados à alimentação devem ser priorizados para aumentar o consumo alimentar dos pacientes.

Entretanto, ao mesmo tempo em que os índices de resto-ingestão apontam para o desperdício, observou-se uma satisfação com os aspectos avaliados durante as enquetes. Os estudos do setor demonstram que, a partir de $85,00 \%$ de satisfação, as unidades que produzem refeições beneficiam-se de uma boa imagem com relação aos seus serviços ${ }^{25}$.

No presente estudo, os pacientes avaliaram positivamente a variedade $(80,00 \%)$, a quantidade $(80,00 \%)$ e a apresentação das refeições $(85,70 \%)$. A maioria desses pacientes recebia dieta hipossódica (74,30\%). Observou-se ainda que esse tipo de dieta obteve um índice de resto-ingestão menor $(33,84 \%)$ em relação à dieta especial com sal (índice de resto-ingestão= $53,68 \%$ ), o que pode revelar que a dieta hipossódica pode estar incorporada ao hábito alimentar desses pacientes, o que implicaria uma menor 
rejeição. Além disso, a consistência alterada das dietas especiais com sal - comumente utilizadas para exames e para preparos de cirurgia, e que impõem aos pacientes modificações temporárias no seu hábito alimentar - pode ter influenciado no índice de resto-ingestão elevado (53,80\%).

A temperatura e o uso de temperos foram os aspectos mais preocupantes, observando-se valores de $43,00 \%$ e $34,30 \%$ respectivamente para os critérios de ruim a regular, o que indica uma possível interferência desses fatores na aceitação dessa dieta.

Donini et al. ${ }^{11}$ revelaram a importância que os pacientes dão ao sabor, à variedade dos alimentos e à forma de apresentação. Wright et al..$^{13}$ observaram que a satisfação com a unidade que produz refeições esteve ligada à variedade, odor, textura da carne, temperatura, sabor da refeição e componentes do cardápio.

Alguns pacientes demonstraram dificuldade em opinar sobre mudanças na alimentação ou rotinas. Corbeau ${ }^{26}$ e Poulain ${ }^{27}$ ressaltam que o contexto da hospitalização transforma os papéis sociais dos indivíduos. A aceitação das refeições e do serviço, nem sempre ideais, é justificada pela pluralidade da população hospitalar. A hospitalização se caracteriza por certa passividade, além do receio de serem mal interpretados ao se queixarem das condições de cuidado e da alimentação.

\section{O N C L U S Ã O}

Os índices de resto-ingestão encontrados (36,09\%) estiveram acima do aceitável para uma população enferma (20,00\%), o que demonstrou uma perda considerável de alimentos. A temperatura e o uso de temperos foram os aspectos de menor satisfação para os pacientes. A partir dos resultados, observou-se a necessidade de avaliação dos processos envolvidos na produção dessas refeições e de elaboração de estratégias que estimulem a ingestão alimentar do paciente, com base não apenas nas funções nutricionais da alimen-tação hospitalar, como também na hedônica e convivial. O presente estudo, como parte de uma proposta de implementação de ações humanizadoras na alimentação hospitalar, pode abrir espaço para outras análises a partir do desenvolvimento dessas ações. A implantação de refeitórios coletivos, o aprimoramento da qualidade sensorial com formação de copeiras e cozinheiras, o uso de utensílios mais adequados para manutenção da temperatura e oficinas culinárias para pacientes podem servir de temas para futuras análises. Vale ressaltar que o estudo não considerou as refeições individuais, o que permitiria identificar casos específicos de pacientes que deixam muitos restos. Na análise realizada, pôde-se apenas indicar os percentuais globais de restos segundo os dias analisados. Portanto, sugere-se que outra análise leve em consideração o monitoramento das refeições individualmente.

\section{A GRADECIMENTOS}

Às acadêmicas do curso de graduação em nutrição da Universidade Federal de Santa Catarina Bruna Fariello Interlandi, Gabriela Martini e Tuani Ferreira, pelo auxílio na coleta de dados.

\section{COLABORADORES}

A.A. SOUSA orientou o desenho do estudo, a coleta e a análise dos dados e participou da redação do artigo. M.S. GLÓRIA e T.S. CARDOSO elaboraram o projeto, realizaram a coleta e a análise dos dados e, igualmente, redigiram o artigo.

\section{REFERÊ NCIAS}

1. Gottraux S, Maisonneuve N, Gevaux D, FonzoChriste C, Chiki M, Guinot-Bourquin S, et al. Screening and treatment of malnutrition: European Council Resolution and its potential application in Switzerland. Rev Med Suisse. 2004; 124(10):617-23.

2. Pérez JIU, Picón CMJ, Benvent EG, Alvarez EM. Detección precoz y control de la desnutrición hospitalaria. Nutr Hosp. 2002; 17(3):139-46.

3. Waitzberg DL, Waleska T, Caiaffa MD, Correia ITD. Hospital malnutrition: the Brazilian National Survey 
294 | A.A. SOUSA et al.

(IBRANUTRI): a study of 4000 patients. Nutrition. 2001; 17(7/8):553-80.

4. Garcia RWD. A dieta hospitalar na perspectiva dos sujeitos envolvidos em sua produção e em seu planejamento. Rev Nutr. 2006; 19(2):129-44. doi: 10.1590/S1415-52732006000200001.

5. Allison A. Hospital food as a treatment. Maidenhead: BAPEN; 1999.

6. Nonino-Borges CB, Rabito El, Silva K, Ferraz CA, Chiarello PG, Santos JS, et al. Desperdício de alimentos intra-hospitalar. Rev Nutr. 2006; 19(3):349-56. doi:10.1590/S1415-527320060030 0006.

7. Almdal T, Viggers L, Beck AM, Jensen K. Food production and wastage in relation to nutritional intake in a general district hospital: wastage is not reduced by training the staff. Clin Nutr. 2003; 22(1): 47-51.

8. Barton $A D$, Beigg $C L$, MacDonald IAP, Allison S. High food wastage and low nutritional intakes in hospital patients. Clin Nutr. 2000; 19(6):445-49.

9. Wilson A, Evans S, Frost G. A comparison of the amount of food served and consumed according to meal service system. J Hum Nutr Dietet. 2000; 13(4):271-75.

10. Kondrup J. Can food intake in hospitals be improved? Clin Nutr. 2001; 20(Supplement 1): 153-60.

11. Donini LM, Castellaneta E, Magnano L, Valerii B, De Felice MR, De Bernardini L, et al. The quality of a restaurant service at a geriatric rehabilitation facility. Ann Ig. 2003; 15(5):583-600.

12. Stanga Z, Zurfluh $Y$, Roselli M, Sterchi AB, Tanner B, Knecht G. Hospital food: a survey of patients' perceptions. Clin Nutr. 2003; 23(3):241-6.

13. Wright ORL, Conelly LB, Capra S. Consumer evaluation of hospital foodservice quality: an empirical investigation. Int J Health Care Qual Assur. 2006; 19(2):181-94.

14. Dupertuis YM, Kossovisky MP, Kyle UG, Raguso CA, Genton I, Pichard CL. Food intake in 1707 hospitalized patients: a prospective comprehensive hospital survey. Clin Nutr. 2003; 22(2):115-23.

15. Lassen KO, Kruse F, Bjerrum M. Nutritional care of Danish medical inpatients: patients' perspectives. Scand J Caring Sci. 2005; 19(3):259-67.
16. Watters CA, Sorensen J, Fiala A, Wismer W. Exploring patient satisfaction with foodservice through focus groups and meal rounds. J Am Diet Assoc. 2003; 103(10):1347-49.

17. Barbosa MFP, Souza TT, Carneiro JM, Sousa AA. Do cuidado nutricional ao cuidado alimentar: percepção de pacientes sobre a refeição hospitalar. Nutr Pauta. 2006; 79:48-54.

18. Helman CG. Cultura, saúde e doença. São Paulo: Artmed; 2003.

19. Poulain JP, Saint-Sevin B. La restauration hospitalière. Toulouse: Cristal; 1990.

20. Proença RPC, Sousa AA, Veiros MB, Hering B. Qualidade nutricional e sensorial na produção de refeições. Florianópolis: UFSC; 2005.

21. Gandra YR. Avaliação de serviços de nutrição e alimentação. São Paulo: Sarvier; 1983.

22. Castro MDAS, Oliveira LF, Silva LPRB. Resto-Ingesta e aceitação de refeições em uma Unidade de Alimentação e Nutrição. Hig Aliment. 2003; 17(114/115): 24-8.

23. Zazzo JF, Puissant MC, Aubert P. Nutritional status and food intake in adult, pediatric and elderly hospitals: a 4-days survey in Assistance PubliqueHôpitaux de Paris. Nutr Clin Metabol . 2003; 17(4 ): 213-17.

24. Campos MTFS, Monteiro JBR, Ornelas APRC. Fatores que afetam o consumo alimentar e nutricional do idoso. Rev Nutr. 2000; 13(3):157-65. doi: 10.15 90/S1415-52732000000300002.

25. Brasil. Ministério da Saúde. Reforma do sistema da atenção hospitalar brasileira. Brasília: Secretaria de Atenção à Saúde; 2004. Série B: Textos Básicos de Saúde, Cadernos de Atenção Especializada.

26. Corbeau JP. S'alimenter à I'hôpital: les dimensions caches de la commensalité. In: Canesqui AM, Garcia RWD. Antropologia e nutrição: um diálogo possível. Rio de Janeiro; Fiocruz; 2005. p.227-38.

27. Poulain JP. Etat des lieux: Comité de Liaison Alimentation Nutrition. In: Ricour C. Mise em place d'une politique nutritionnelle dans les etablissements de santé. Paris: Ministere de la Santé DHOS; 2002.

Recebido em: 21/6/2009

Versão final reapresentada em: 30/10/2009 Aprovado em: 12/5/2010 